\title{
LA PSICOTERAPIA RELACIONAL INTEGRATIVA EN EL CAMPO DE LA DISCAPACIDAD INTELECTUAL
}

\section{INTEGRATIVE RELATIONAL PSYCHOTHERAPY IN THE FIELD OF INTELLECTUAL DISABILITY}

\author{
Verónica Aguado Rodríguez \\ Aukebi Centro Psicológico, Bilbao, Bizkaia, España \\ ORCID: https://orcid.org/0000-0002-8137-5546
}

Cómo referenciar este artículo/How to reference this article:

Aguado Rodríguez, V. (2021). La psicoterapia Relacional Integrativa en el campo de la Discapacidad Intelectual. Revista de Psicoterapia, 32(118), 163-175. https://doi.org/10.33898/rdp.v32i118.491

\begin{abstract}
Resumen
Tradicionalmente, la intervención psicoterapéutica en el campo de la discapacidad intelectual, ha recibido poca atención por los diferentes desafíos que implica el trabajo con personas con dicha diversidad. Elementos tales como la diversidad en el estilo cognitivo, dificultades de lenguaje y/o comunicación y estereotipos en torno a la discapacidad intelectual, entre otros, han hecho que su práctica sea limitada, y más aún su estudio. El objetivo del presente artículo es realizar una breve aproximación al ámbito de la discapacidad intelectual desde el modelo de la Psicoterapia Relacional Integrativa basada en el desarrollo evolutivo (PRI). Para ello, se presentarán breves casos clínicos con el fin de mostrar y acercar de forma práctica parte de la teoría, filosofía e intervención del modelo PRI.

Palabras clave: psicoterapia, psicoterapia integrativa, discapacidad intelectual, relación terapéutica, desarrollo evolutivo, métodos
\end{abstract}

\begin{abstract}
In the field of the intellectual disability, psychotherapeutic intervention is not completely well characterized. Cognitive style, language limitations, communication difficulties and stereotypes around the intellectual disability are among the main causes that do not facilitate the study and application of psychotherapy. This article represents an attempt to use Integrative Relational Physiotherapy (IRP) based on the evolutive development as a tool to study and treat the intellectual disability. In order to present part of the theory, philosophy and intervention method of this IRP model, several clinical cases are presented.

Keywords: psychotherapy, integrative psychotherapy, intellectual disability, therapeutic relationship, evolutive development, methods
\end{abstract}


Históricamente, el término de discapacidad intelectual (DI) se ha conocido como retraso mental, sin embargo, desde el 2004 existe un consenso internacional a favor del concepto DI con el fin de eliminar el estigma y devaluación que conlleva dicho concepto (Marquéz-Caraveo et al., 2011). Si bien, a partir del año 2015 diferentes foros de personas con DI y entidades sociales proponen utilizar el concepto "diversidad funcional intelectual" con el objetivo de perfilar el lenguaje y mostrar una realidad más amplia del funcionamiento cognitivo de las personas, aún hoy en día no está establecido como tal, por lo que en el presente artículo se utilizará el término DI. En todo caso, ambos conceptos aluden a un funcionamiento general cuya definición, según la asociación americana de discapacidad intelectual y del desarrollo (AAIDD, 2010/2011), sería la siguiente: "la discapacidad intelectual se caracteriza por limitaciones significativas tanto en la funcionamiento intelectual, como en la conducta adaptativa, tal y como se ha manifestado en habilidades adaptativas, conceptuales y prácticas. Esta discapacidad se origina antes de los 18 años”.

Las personas con DI presentan un proceso más lento e incompleto de lo habitual en la adquisición de las habilidades neurocognitivas durante el desarrollo evolutivo (Di Nuovo y Buono, 2007). Esto implica que pueden tener dificultades para la comprensión, el aprendizaje, recordar cosas nuevas y otras funciones cognitivas, que se manifiestan durante el desarrollo y que contribuyen al nivel de inteligencia general, generando dificultades para la vida cotidiana, comunicación y socialización (Ke y Liu, 2017; Marquéz-Caraveo et al., 2011).

Teniendo en cuenta la realidad funcional interna y externa que presentan las personas con DI, la comorbilidad con algunos trastornos psicológicos es una complicación común, presentando de este modo una mayor incidencia de problemas en la salud mental en comparación con población que no presenta una DI (Di Nuovo y Buono, 2007; Galí, 2018; Martorell et al., 2009; Turygin et al., 2014).

Sin embargo, a pesar de esta alta tasa de dificultades en la salud mental, la psicoterapia en el campo de la DI ha tendido a ser una disciplina terapéutica poco utilizada, y casi nada estudiada. Tradicionalmente, la salud mental de las personas con DI ha sido atendida desde las farmacoterapias y las técnicas de modificación de conducta (Garvía y Flórez, 2018). Si bien, es importante tener en cuenta ambas terapias a la hora de intervenir sobre la salud mental, en las personas con DI generalmente, se ha prestado menos atención a los procesos emocionales y fenomenológicos de la persona; es decir, a la propia construcción subjetiva individual desarrollada a través de las experiencias vitales. Y en muchas ocasiones, los conflictos psicológicos derivados de esas experiencias vitales, quedan ocultos bajo la propia DI, sin ni siquiera valorar la opción de poder realizar un proceso psicoterapéutico.

Probablemente, uno de los principales motivos sea debido a la creencia general de que los tratamientos psicológicos son poco eficientes en dicha población debido a los déficits cognitivos y a las limitaciones de comunicación que habitualmente presentan (Galí, 2018). Sin embargo, la dificultad radicaría en la no adaptabilidad de los métodos psicoterapéuticos y no tanto en las personas con DI. De ahí, que estos 
métodos requerirían de capacidad de adaptación en el estilo, en las técnicas y en el ritmo de la intervención, así como una mirada amplia por parte del psicoterapeuta.

Aunque, en la última década cada vez se tiene más presente la psicoterapia como opción de intervención en este campo debido al aumento de su demanda, y debido a que cada vez hay más expertos que aportan sus beneficios (e.g., Brown et al., 2011; Drury y Alim, 2014; Galí, 2018; Garvía y Flórez, 2018; McGuire y Chicoine, 2006/2010), aun hoy en día es escasa la literatura en torno a ello. Por ello, la finalidad del presente artículo es presentar una breve aproximación teórica desde el marco de la Psicoterapia Relacional Integrativa y la forma en la que este modelo teórico ofrece una mirada completa, compleja, coherente y humanista para la intervención psicoterapéutica con personas con DI. El contenido se centra en tres ejes relevantes vinculados a este marco teórico: la relación terapéutica, la perspectiva evolutiva y la teoría de los métodos, intercalando pequeños fragmentos de casos clínicos a modo de ejemplos básicos ${ }^{1}$ y prácticos.

\section{Psicoterapia Relacional Integrativa Basada en el Desarrollo Evolutivo}

La Psicoterapia Relacional Integrativa (PRI) fue desarrollada por Richard. G. Erskine (PhD) en los años 70 y 80 con la finalidad de dar respuesta a la necesidad de aunar e integrar de forma coherente diferentes modelos teóricos y métodos de intervención para el abordaje en la psicoterapia de los sistemas afectivos, cognitivos, conductuales, fisiológicos y relacionales de las personas (Erskine y Moursund, 1988/2014; O’Reilly-Knapp, 2016). Por ello, la PRI tiene en cuenta muchos enfoques teóricos, tales como el psicodinámico, el enfoque centrado en la persona, el cognitivo-conductual, la teoría de la Gestalt, teorías sistémicas y el análisis transaccional, entre otros (Erskine y Moursund, 1988/2014). Cada uno de ellos proporciona una explicación válida del funcionamiento psíquico y comportamental del ser humano en el que se complementan, al integrarse de forma coherente entre sí, dando lugar a un amplio modelo con una base compleja y coherente con una teoría de la motivación, teorías de la personalidad y teoría de los métodos (Erskine, 2015/ 2016; Erskine y Moursund, 1988/2014; Erskine et al., 1999/2012).

Además, “integrativa” también hace referencia al proceso de integración de la personalidad que se da en el proceso de la psicoterapia, es decir, asimilar aspectos "fragmentados", no conscientes o no resueltos de uno mismo y hacerlos parte de una personalidad cohesionada, con el fin de reestablecer la capacidad del contacto interno y externo, reduciendo así el uso de estrategias auto-protectoras que inhiben la espontaneidad y limitan la flexibilidad en la resolución de problemas, el mantenimiento de la salud y las relaciones saludables (Erskine, 2015/2016; Erskine et al., 1999/2012; Erskine y Moursund, 1988/2014).

Tanto en la teoría como en los métodos existen principios y valores con respecto al valor del ser humano, la importancia de la calidad de la vida, el significado de las relaciones, y el apoyo del crecimiento psicológico a través de una psicoterapia relacional (O’Reilly-Knapp, 2016). Estos valores se presentan por medio de los 
principios filosóficos y sirven como cimientos sobre los que se construye la PRI (Erskine, 2015/2016). Se nombran a continua
A) Todas las personas son igualmente valiosas
B) Toda experiencia humana se organiza física, afectiva y/o cognitivamente
C) Toda conducta humana tiene significado en algún contexto
D) El contacto interno y externo es esencial para el funcionamiento humano
E) Todas las personas buscan relaciones y son interdependientes a lo largo de la vida
F) Los seres humanos tienen el impulso innato de crecer y desarrollarse
G) Los seres humanos sufren interrupciones relacionales, no "psicopatología”.
$\mathrm{H})$ El proceso intersubjetivo en psicoterapia es más importante que el conte- nido de la psicoterapia

\section{La Relación Terapéutica}

Desde este modelo teórico, se atienden las diferentes dimensiones de la persona (afectiva, cognitiva, comportamental y fisiológica) dentro de un marco relacional. Por ello, la relación terapéutica que se crea entre el psicoterapeuta y la persona es un eje principal y esencial a lo largo de todo el proceso psicoterapéutico, y si esta relación es sintónica con la persona con la que se trabaja, proporcionará la oportunidad de intervenir en los patrones relacionales inconscientes que conforman el sistema automatizado de la organización mental (memorias implícitas y procedimentales, reacciones fisiológicas y emocionales de supervivencia, modelos operativos internos, etc.), creando y adquiriendo una experiencia alternativa y reparadora del malestar y daño intrapsíquico y relacional (Criswell y Erskine, 2015; Erskine et al., 1999/2012). La teoría de motivación en la cual se sustenta la PRI y la relevancia de la relación terapéutica, está construida en base a los imperativos biológicos del “impulso del ser” descritos por Berne (citado en O’Reilly-Knapp y Erskine, 2003) y conocidos como "hambres": hambre de estímulo, hambre de estructura y hambre de relación. Todo ser humano está repleto de estímulos tanto internos como externos y nunca se está sin ellos, es un sistema informativo que promueve la satisfacción de las necesidades internas y externas (O’Reilly-Knapp y Erskine, 2003). Estos estímulos requieren de estructura para darles un sentido, comprensión y una capacidad de lenguaje (O’Reilly-Knapp, 2016; O’Reilly-Knapp y Erskine, 2003), es decir, la necesidad de organizar la experiencia. Y asimismo, el procesamiento del sistema de información de los estímulos y la estructuración, se dan siempre dentro de la relación como seres sociales que somos (Erskine, 2015/2016; Erskine et al., 1999/2012; Martínez, 2017). Son tres "hambres" que se dan de forma simultánea, se interconectan, se retroalimentan y se compensan entre sí. Si estas necesidades no son cubiertas de forma suficiente, generarán un desajuste en el sistema interno de la persona a fin de crear un ajuste adaptativo, sin embargo, pudiendo llevar a la persona a desarrollar conflictos emocionales, relacionales, comportamentales y de pensamiento. 


\section{Ejemplo 1}

Ane (12 años) era una joven con síndrome de Down y diagnóstico de TEA (trastorno del espectro autista). Uno de los motivos de consulta que presentó la familia fue que habían observado que, cada vez más a menudo, Ane realizaba una serie de rituales repetitivos y que se quedaba "bloqueada" (paralizada y sin reacción) en situaciones cotidianas tales como la compra en el supermercado, en el patio del instituto y en el cine. Una de las hipótesis formuladas tras la valoración inicial, fue que Ane atravesaba un periodo muy "estimulante" (cambios corporales y neuronales por la propia etapa evolutiva, cambio de instituto y grupo de referencia, muchas actividades extraescolares, etc.) que podría estar resultando difícil de procesar por ella. Desde esta premisa, y colocando la mirada en la teoría de la motivación, una de las intervenciones que se trabajó, en este caso con la familia, fue el reajuste de la "sobreestimulación”. Se procuró, como punto de partida, que tuviera más espacio para el descanso (dando la oportunidad de que pudiera integrar y estructurar internamente sus vivencias) y el fomento de encuentros relacionales con personas de confianza y nutritivas para ella, además de disminuir las exigencias y/o situaciones de estrés del día a día. Esta comprensión desde la teoría de la motivación ayudo a su familia a colocar una mirada diferente a las conductas y respuestas de Ane, pudiendo ajustarse un poco más a sus necesidades. En pocas semanas los rituales y los bloqueos disminuyeron significativamente en ella. Paralelamente, se trabajó con Ane la seguridad en la relación terapéutica, poniendo especial atención a su ritmo interno y a la sintonía evolutiva y afectiva. A través de técnicas proyectivas y activas, entre otros aspectos, se pudo trabajar en alternativas de autorregulación, resignificación de las anticipaciones que aparecían en forma de fantasías (es decir, a comprender el significado de las anticipaciones para poder diferenciar realidad-fantasía, y darles un nuevo valor más actualizado al contexto) y el concepto de identidad.

A través de la perspectiva de la teoría de la motivación, existen una serie de necesidades específicas y esenciales en torno al contacto interpersonal que se denominan necesidades relacionales (Erskine et al., 1999/2012; O’Reilly-Knapp y Erskine, 2003). Las necesidades relacionales son tales como la necesidad de sentir racional, emocional y visceralmente la seguridad en la relación, de sentirse validado y significado en la experiencia interna y externa a través de la relación, de tener una relación en la cual sentirse aceptado y dependible de alguien estable y confiable, de poder autodefinirse libremente, de hacer impacto en otra persona, de sentir la iniciativa del otro hacía uno mismo, de compartir y confirmar experiencias similares y de ser capaces de mostrar afecto y aprecio en la relación. Del mismo modo, si estas necesidades no son cubiertas de forma persistente crearán en la persona una sensación de desajuste incrementando la intensidad de la necesidad y se experimentarán fenomenológicamente como un anhelo, un vacío, una soledad persistente y/o como un intenso impulso, acompañado a menudo de agitación interna (Erskine, 2015/2016). Por ello, una de las tareas principales que corresponde en la 
intervención psicoterapéutica desde el modelo PRI es atender a estas necesidades relacionales, entre otras formas, desde la relación terapéutica.

\section{Ejemplo 2}

Aritz, un joven de 16 años con síndrome de Down, acudió a psicoterapia a través de la demanda de sus padres porque tenía reacciones de enfado "desmesuradas y agresivas". Aritz era una persona muy curiosa, con una capacidad sensitiva alta y muy perceptivo a la emocionalidad de los otros, además le encantaban los cómics, escuchar música y salir con sus amigos. Su estilo relacional en la consulta era amable, pausado y muy respetuoso y sereno. Resultaba difícil imaginarlo con reacciones de enfado intensas y/o agresivas. Sin embargo, a medida que la psicoterapia avanzaba, no sólo hablaba de ello, sino que también, comenzó a reproducir esas reacciones cuando conectaba con situaciones que experimentaba como injustas. Estas situaciones experimentadas como injustas eran muy diversas, se le activaba la misma intensidad emocional cuando conectaba con los recuerdos relacionados a situaciones como que no hubiera un cómic que buscaba en la tienda, como cuando conectaba con el acoso que sufrió en el colegio. Expresaba literalmente "nadie me toma en serio, es injusto, porque tengo Down, pues yo doy puñetazos y así saben quién soy", "Yo voy a defender a los pequeños, soy un héroe ¡como Batman! Mis puños son la justicia" "si soy un héroe no saben que tengo síndrome de Down". A través de la indagación sintónica, su narrativa y la escucha "de las necesidades relacionales", se pudo dar un significado al enfado más allá de una reacción "desajustada”. A través de la relación psicoterapéutica pudo sentir el impacto de su dolor, la confirmación y validación de su experiencia, la posibilidad de autodefinirse más allá del síndrome de Down y, por supuesto, la seguridad de poder conversar e intervenir sobre el sentimiento de vergüenza que se le activa cuando conecta con el síndrome de Down y la mirada de los demás. Esto le permitió ampliar el nivel de conciencia sobre sí mismo, desarrollando otras respuestas más ajustadas a la realidad y sobre todo a sus necesidades relacionales.

\section{Perspectiva Evolutiva}

Si uno de los ejes esenciales para la intervención desde este marco teórico es lo relacional y la relación psicoterapéutica, otro de los ejes que marca una diferencia importante es intervenir a través de la perspectiva evolutiva. Tener esta perspectiva implica ser sensibles y tener capacidad de sintonizar con las necesidades que emergen a través de pequeñas regresiones de edad y expresiones a través de la transferencia que surgen en el proceso psicoterapéutico (Erskine, 2019). Para ello, se requiere de un conocimiento profundo sobre las teorías del desarrollo evolutivo, entre ellas, las teorías de apego de Bowlby (Ainsworth y Bowlby, 1991; Bowlby, 1973, 1979/1986), las teorías del desarrollo cognoscitivo de Piaget y Vygotsky (citados en Raynaudo y Peralta, 2017), las teorías del desarrollo de Erikson (Erikson, 1982/2000) y las teorías de mentalización de Fonagy (Bateman y Fonagy, 
2016/2016; Sánchez-Quintero y De la Vega, 2013), y comprender las respuestas concretas que cada necesidad evolutiva requiere del psicoterapeuta. Cada persona con la que se trabaja presenta un estado de desarrollo evolutivo específico en las diversas dimensiones de su funcionamiento humano, que pueden ir o no acorde con la edad cronológica, y éstas además, se presentan de forma dinámica como respuesta a momentos determinados en los que se activan recuerdos, memorias y/o procesos implícitos ante situaciones de estrés y/o conflicto. Tal como indican Erskine y Mauriz-Etxabe (2020):

Esto requiere que pensemos sobre nuestros clientes desde una perspectiva evolutiva con sensibilidad hacia las necesidades físicas y relacionales de la niña o el niño, los posibles conflictos relacionales y las pérdidas que pudo haber sufrido, así como en la calidad y tipo de influencia proporcionada por sus cuidadores y, finalmente, en las estrategias que encontró para lograr una estabilidad, algún tipo de regulación e incluso para mejorar y evolucionar. (p. 206)

Estas estrategias son las que hoy en día en momentos de estrés, conflicto y/o crisis, probablemente se activen con el fin de procurar restablecer la estabilidad interna y externa. La mirada de la persona psicoterapeuta hacía esta perspectiva evolutiva posibilitará intervenir, a través de una relación terapéutica reparadora, sobre aquellas necesidades vinculadas a crisis arcaicas pero ligadas al hoy. Tener una perspectiva evolutiva no significa "infantilizar" a la persona con DI, significa comprender en profundidad su funcionamiento interno y adaptarse a su estilo relacional, cognitivo, de comunicación, etc., con el máximo respeto, no olvidando a la persona que se tiene delante y su etapa evolutiva vincula a su edad cronológica, salvaguardando y fomentando todo el derecho al desarrollo de su propia autonomía personal.

\section{Ejemplo 3}

Leticia (persona con DI) tenía 21 años cuando su padre falleció por una enfermedad que padecía desde hacía un año. Según su madre, desde algo antes de fallecer su padre, Leticia comenzó a tener comportamientos "extraños" para su edad, "como si de repente se hubiera convertido en una niña pequeña". Se chupaba el dedo pulgar de forma repetitiva, su lenguaje verbal había cambiado en el tono y en el vocabulario como si hubiera habido un retroceso, volvió a emerger la enuresis nocturna que tuvo durante unos años en su infancia y su estilo relacional se volvió más dependiente e inseguro. Comprender su estado como una regresión a modo de respuesta a un intenso miedo y tristeza derivado de la vivencia de una pérdida tan vital para Leticia, permitió poder acompañarla en la elaboración del duelo desde la sensibilidad a sus necesidades más primarias que emergieron como una forma de estabilización y regulación interna. Para ello fue importante trabajar en la seguridad de la relación terapéutica, la sintonía evolutiva a través del tono de voz y los movimientos corporales siendo suaves y cuidadosos, el ritmo de las 
intervenciones también con la delicadeza de no ser de "más” respetando su propio ritmo interno. Esto permitió que ella poco a poco pudiera ir conectando con el dolor de la pérdida, el miedo a lo que les iba a ocurrir ahora que no estaba aita, y aquellos aspectos que se quedaron sin resolver en la relación con su padre. Sobre todo con una de las fantasías "secretas" que tenía que ver con escenas de enfado con su padre, en las que él le decía "me vas a acabar matando". En ella, internamente se representaba esta frase como una realidad, generando un gran sentimiento de culpa por “crear su enfermedad” y vergüenza, por lo que se había convertido en un secreto "no puede saberlo nadie si no voy a la cárcel”. Esto requirió mucha intervención respetuosa y cuidadosa hasta que fue comprendiendo estas fantasías e integrando todas las emocionales y sensaciones que se habían activado.

\section{Los Métodos en la Psicoterapia Relacional Integrativa}

Como ya se ha mencionado previamente, la teoría de los métodos se basa en la idea de que la cura se da a través de una relación psicoterapéutica de pleno contacto, en donde se enfatiza el contacto interno a través de los procesos intrapsíquicos, y el contacto externo a través de los proceso interpersonales (Erskine, 2015/2016). Los métodos están compuestos por tres categorías principales: indagación, sintonía e implicación, y cada una de ellas son centrales para la relación terapéutica y para la reorganización de la personalidad que reconduce al bienestar emocional y psicológico (Erskine et al., 1999/2012; Erskine y Trautmann, 1996; O’Reilly-Knapp y Erskine, 2003).

\section{Indagación}

Erskine et al. (1999/2012) describen detalladamente la función de la indagación como una forma de intervención dirigida a acompañar y ayudar a la persona a extender su propia conciencia sobre los pensamientos, sentimientos, sensaciones y percepciones, especialmente por medio de la indagación fenomenológica. Esto permite crear una nueva imagen de sí misma, de los demás y de la vida (Erskine y Trautmann, 1996; Martinez, 2017). Además, la indagación ayuda al psicoterapeuta a salir de un rol interpretativo, colocándolo en un rol de curiosidad, interés y respeto por la experiencia única de la persona (Martínez, 2017). Va más allá de hacer preguntas, significa tener la asunción de que el psicoterapeuta "no sabe nada" sobre la experiencia subjetiva y la conceptualización del otro.

\section{Ejemplo 4}

Pedro (con síndrome de Down) de 38 años acudió a consulta porque únicamente quería estar solo en su habitación. Su familia estaba muy preocupada por ello, él en cambio no estaba preocupado por eso, a él le preocupaba conocer y comprender a su "familia de antepasados". Pedro presentaba rasgos psicóticos a través de sus fantasías que, sin llegar a estar desajustado y con un buen nivel de funcionalidad (tenía tratamiento farmacológico), en el último periodo le habían llevado a estar 
más retirado, porque tenía el proceso obsesivo muy activado con la idea que él en realidad nació en otra familia de origen noruego. Durante muchas sesiones la finalidad principal fue escuchar atentamente aquello que le preocupaba, indagar sobre su "familia de antepasados", qué significaban para él, de qué forma los había “dibujado en su mente”, cuál era la historia que albergaba, en qué le iba ayudar conocerles y comprenderles... En definitiva, desgranar aquellas fantasías y tomar conciencia de las necesidades que había en ellas. Un día en una sesión dijo "ojalá mi padre fuera como Peter (padre de la familia de antepasados de la fantasía)". Su padre había fallecido hacía dos años. Este contacto permitió abrir una línea de trabajo psicoterapéutico sobre la relación que vivió con su padre, en la que él sentía "no me quería porque yo era Noruego". Al indagar sobre si ser noruego era "como" tener síndrome de Down, él dijo que no, pero que muy parecido. De nuevo, esto abrió nuevos caminos en el proceso de psicoterapia.

\section{Sintonía}

La sintonía implica un proceso que requiere, por un lado, de la sensibilidad y la capacidad de reconocimiento e identificación con las sensaciones, necesidades y sentimientos de la otra persona y, por otro, de la transmisión y comunicación de dicha sensibilidad, creando así un proceso de comunión y de contacto interpersonal único (Erskine et al., 1999/2012; Erskine, 2015/2016; Erskine y Trautmann, 1996). La sintonía no se da sólo a través del canal verbal, se requiere también, la apertura de los canales sensorio-perceptivos, la comunicación afectiva y la resonancia corporal; y por ello, la postura corporal, los movimientos, el tono de voz y los gestos faciales del psicoterapeuta, son elementos esenciales para transmitir la sintonía, y con ello validar las necesidades y los sentimientos, y la importancia de todo ello (Erskine et al., 1999/2012; Martínez, 2017). El proceso de sintonización procura una sensación de seguridad y estabilidad que permite a la persona experimentarse a sí misma de forma diferente, creando nuevas conexiones y representaciones internas (Erskine y Trautmann, 1996; Bateman y Fonagy, 2016/2016).

La sintonía será diferente en base a la resonancia producida y a la reciprocidad necesaria para crear un espacio de contacto en la relación. Según estas diferencias, la sintonía se puede categorizar en: sintonía rítmica, sintonía cognitiva, sintonía evolutiva, sintonía afectiva y sintonía de las necesidades relacionales (Erskine, 2015/2016; Erskine et al., 1999/2012; Erskine y Trautmann, 1996; O’Reilly-Knapp y Erskine, 2003), sin embargo, todas ellas se entrelazarán en el proceso relacional produciendo una sintonización dinámica.

Dentro del campo de la DI, aunque todos los estilos de sintonización son fundamentales, probablemente la sintonía cognitiva y evolutiva tienen una relevancia especial, debido a que los estilos personales pueden ser tan diferentes que la sintonización realmente se convierte en un reto. 


\section{Ejemplo 5}

Martín (25 años con síndrome de Down y diagnóstico de psicosis desde la infancia) tenía un estilo cognitivo único. Sus pensamientos y, por lo tanto, también parte de su comunicación, se vinculaban y se elaboraban a través de las historias Disney, cuentos infantiles o la saga de Harry Potter. Fue muy complicado crear una sintonía cognitiva con la cual comprender en profundidad el mecanismo y el procedimiento interno de sus pensamientos. Sin embargo, a través de la sintonía afectiva fue más fácil. Con el tiempo, gracias a la indagación respetuosa y sintónica, el significado de las historias y la metáfora en ellas fueron revelándose en el proceso de psicoterapia. Una de ellas fue a través de la historia de la Bella y la Bestia de Disney, en la que él era la Bestia y durante varias sesiones solo habló y se relacionó como Bestia. A través de esta historia se realizó un importante trabajo de autodefinición.

\section{Implicación}

La implicación hace referencia a la actitud del psicoterapeuta ante la persona. Como indican Erskine et al. (1999/2012) "la indagación trata de lo que hace un psicoterapeuta; la implicación no trata tanto de hacer como de ser" (p. 135). Significa poder estar plenamente presente desde una autenticidad real. Erskine (2015/2016) también afirma que "la implicación enfatiza el reconocimiento, la validación, la normalización y la presencia hacia la persona, pudiendo disminuir así la descalificación interna que forma parte de un proceso defensivo arcaico” (p.20). Así como “despatologizar” y aumentar la comprensión funcional de los procesos internos y externos (Erskine, 2015/2016).

\section{Ejemplo 6}

Celia tenía 30 años y una parálisis parcial física y DI leve derivada de una falta de oxígeno durante su nacimiento. Durante toda su vida se ha sentido "insignificante, sin que a nadie le importe. No hay nadie para mí”. Para este proceso terapéutico fue esencial mostrar y hacer sentir la presencia psicoterapéutica, ella la buscaba constantemente y la ponía a "prueba” buscando los límites (llamadas constantes, emails, enfados, etc.). Si bien, crear una sólida estructura a través de los límites fue necesario, mostrar la disponibilidad emocional y presencial, también. Tomar en serio sus enfados, atender ciertas llamadas y ciertos emails, establecer límites protectores y atender la contratransferencia fueron, entre otros elementos, formas en las que la presencia psicoterapéutica fue tomando forma en las sesiones y fuera de ellas, creando un vínculo terapéutico sólido y estable.

Estas categorías de los métodos se representan gráficamente en el diagrama del Keyhole u Ojo de la Cerradura mostrado en la figura 1. Este diagrama, que es expuesto en profundidad por Erskine et al. (1999/2012), pretende recoger los métodos específicos y la idea sobre cómo la indagación, la sintonía y la implicación están inevitablemente interconectadas en la relación psicoterapéutica. De forma 
concreta, el diagrama representa la sintonía como la base que guía la indagación y que determina la naturaleza de la implicación terapéutica.

Figura 1. Keyhole (Erskine et al., 1999/2012; O’Reilly-Knapp y Erskine, 2003)
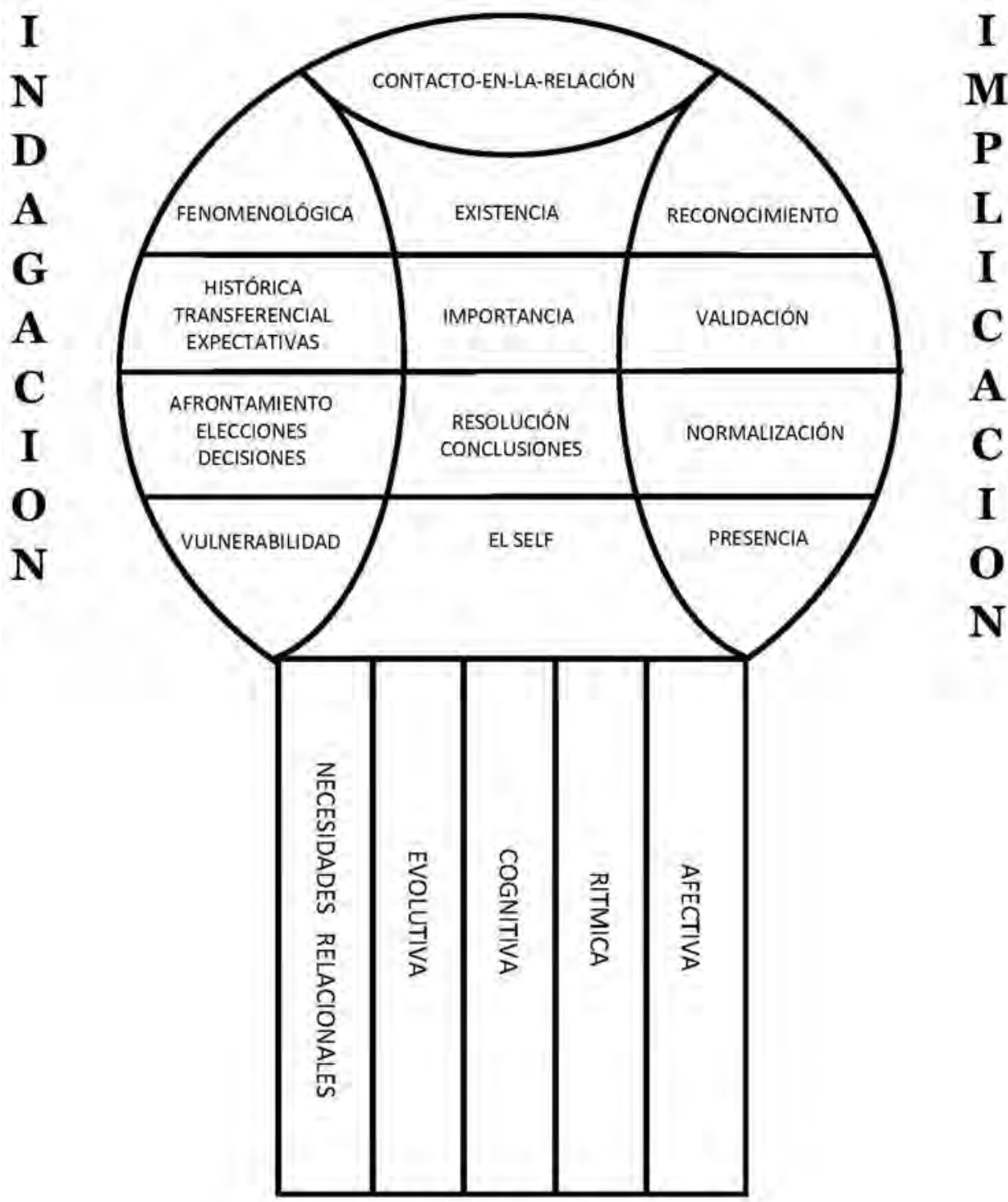

SINTONIA 


\section{Conclusión}

La psicoterapia no ha sido una disciplina terapéutica muy utilizada en el campo de la DI, a pesar de las evidencias sobre su eficacia en la mejora de la salud mental. Establecer marcos teóricos en torno a ello se presenta como un desafío necesario y actual. Desde esta premisa, este artículo describe una aproximación al modelo teórico de la PRI como un marco referencial sólido y robusto para la intervención psicoterapéutica. Además, este modelo, a través de la mirada teórica que brinda y sus principios filosóficos como marco de referencia, permite comprender en profundidad a la persona (y en este caso a la persona con DI) por medio de sus conductas, fantasías, respuestas emocionales y fisiológicas, estilo relacional y cognitivo,... y construir desde la relación terapéutica un "mapa” para poder comprender sus necesidades, sus experiencias fenomenológicas, y así establecer una intervención sintónica y ajustada a su realidad interna y externa, más allá del propio diagnóstico y/o la discapacidad intelectual.

\section{Notas}

1 Los ejemplos se presentan de forma sucinta, con la finalidad de enfatizar en aquel concepto o idea que se esté presentando. (p. 4).

\section{Referencias}

AAIDD (2011). Discapacidad intelectual. Definición, clasificación y sistemas de apoyo social (M. Verdugo, Trad.). Alianza Editorial. (Trabajo original publicado en 2010)

Ainsworth, M. D. S. y Bowlby, J. (1991). An ethological approach to personality development. American Psychologist, 46(4), 333-341. https://doi.org/10.1037/0003-066X.46.4.333

Bateman, A. y Fonagy, P. (2016). Tratamiento basado en la mentalización para trastornos de la personalidad: Una guía práctica (F. Mora, Trad.). Descleé de Brouwer. (Trabajo original publicado en 2016)

Bowlby, J. (1973). Attachment and loss, vol. II: Separation [Apego y pérdida, vol. II: Separación]. Basic Books.

Bowlby, J. (1986). Vínculos afectivos: formación, desarrollo y pérdida (A. Guera, Trad.). Morata. (Trabajo original publicado en 1979)

Brown, M., Duff, H., Karatzias, T. y Horsburgh, D. (2011). A review of the literature relating to psychological interventions and people with intellectual disabilities: issues for research, policy, education and clinical practice [Una revisión de la literatura relacionada con las intervenciones psicológicas y las personas con discapacidad intelectuales: temas para la investigación, las políticas, la educación y la práctica clínica]. Journal of Intellectual Disabilities, 15(1), 31-45. https://doi.org/10.1177/1744629511401166

Criswell, G. E. y Erskine, R. G. (2015). La psicoterapia del contacto en la relación. Diálogos con Richard Erskine. Revista de Psicoterapia, 26(100), 115-127. http://ojs.revistadepsicoterapia.com/index.php/rdp/article/ view/48/18

Di Nuovo, S. F. y Buono, S. (2007). Psychiatric syndromes comorbid with mental retardation: differences in cognitive and adaptive skills [Síndrome psiquiatríco comórbido con retraso mental: diferencias en habilidades cognitivas y adaptativas]. Journal of Psychiatric Research, 41(9), 795-800. https://doi.org/10.1016/j. jpsychires.2006.02.011

Drury, H. y Alim, N. (2014). Integrative psychotherapy in intelectual disabilities: using cognitive-behavioural and psychodynamic formulations [Psicoterapia integrativa en discapacidad intellectual: utilizando formulaciones cognitivo-comportamentales y psicodinámicas]. Advances in Mental Health and Intellectual Disabilities, 8(3), 197-209. https://doi.org/10.1108/AMHID-10-2012-0001

Erikson, E. H. (2000). El ciclo vital completado(E. Prieta, Trad.). Paidos Ibérica. (Trabajo original publicado en 1982) 
Erskine, R. G. (2016). Presencia terapéutica y patrones relacionales: conceptos y práctica de la Psicoterapia Integrativa (A. Pérez Burgos, Trad.). Karnac. (Trabajo original publicado en 2015)

Erskine, R. G. (2019). Developmentally based, relationally focused Integrative psychotherapy: Eight essential points [Psicoterapia Integrativa basada en el desarrollo y centrada en la relación: Ocho puntos esenciales]. International Journal of Integrative Psychotherapy, 10, 49-59. https://www.integrative-journal.com/index. php/ijip/article/view/144/96

Erskine, R. G. y Mauriz-Etxabe, A. (2020). Inferencia, re-experimentación y regresión: Psicoterapia de los estados niño del Yo. Revista de Psicoterapia, 31(116), 205-226. https://doi.org/10.33898/rdp.v31i116.362

Erskine, R. G. y Moursund, J. P. (2014). La Psicoterapia Integrativa en acción (A. Pérez Burgos, Trad.). Descleé de Brouwer. (Trabajo original publicado en 1988)

Erskine, R. G., Moursund, J. P. y Trautmann, R. L. (2012). Más allá de la empatía. Una terapia de contacto en la relación (N. Maestre, Trad.). Descleé de Brouwer. (Trabajo original publicado en 1999)

Erskine, R. G. y Trautmann, R. L. (1996). Methods of an integrative psychotherapy [Métodos de una psicoterapia integrativa]. Transactional Analysis Journal, 26, 316-328. https://doi.org/10.1177/036215379602600410

Galí, D. (2018). Intervenciones psicológicas en adultos con discapacidad intelectual y trastorno límite de la personalidad comórbido. Revista Española de Discapacidad, 6(2), 49-62. https://doi.org/10.5569/23405104.06.02.03. https://www.cedd.net/redis/index.php/redis/article/view/329/pdf_112

Garvía, B. y Flórez, J. (2018). Psicoterapia y farmacoterapia en la discapacidad intelectual. CEPE.

Ke, X. y Liu, J. (2017). Discapacidad intelectual (M. Irarrázabal, A. Martin, F. Prieto-Tagle y O. Fuertes, Trad.). En J. M. Rey (Ed.), Manual de salud mental infantil y adolescente de la IACAPP (pp. 1-28). Asociación Internacional de Psiquiatría del Niño y el Adolescente y Profesionales Afines. https:/iacapap.org/content/ uploads/C.1-Discapacidad-Intelectual-SPANISH-2018.pdf

Marquéz-Caraveo, M. E., Zanabria-Salcedo, M., Pérez-Barrón, V., Aguirre-García, E., Arciniega-Buenrostro, L. y Galván-García, C. S. (2011). Epidemiología y manejo integral de la discapacidad intelectual. Salud Mental, 34, 443-449. https://www.medigraphic.com/pdfs/salmen/sam-2011/sam115g.pdf

Martínez, J. M. (2017). Relational methods and theories of intersubjectivity [Métodos relacionales y teorías de la intersubjetividad]. International Journal of Integrative Psychotherapy, 8, 1-15. https://www.integrativejournal.com/index.php/ijip/article/view/138/86

Martorell, A., Tsakanikos, E., Pereda, A., Gutiérrez-Recacha, P., Bouras, N. y Ayuso-Mateos, J. L. (2009). Mental health in adults with mild and moderate intellectual disabilities: the role of recent life events and traumatic experiences across the life span [Salud mental en adultos con discapacidad intellectual leve y moderada: el papel de los eventos recientes y experiencias traumáticas a lo largo de la vida]. The Journal of Nervous and Mental Disease, 197(3), 182-186. https://doi.org/10.1097/NMD.0b013e3181923c8c

McGuire, D. y Chicoine, B. (2010). Bienestar mental en los adultos con síndrome de Down. Una guía para la comprender y evaluar sus cualidades y problemas emocionales y conductuales (R. León y J. Flórez, Trad.). Fundación Iberoamericana Down21. (Trabajo original publicado en 2006)

O’Reilly-Knapp, M. (2016). Integrative Psychotherapy 'Revisited' [Psicoterapia Integrativa 'Revisitada']. International Journal of Integrative Psychotherapy, 7, 49-59. https://www.integrative-journal.com/index. php/ijip/article/view/126/79

O’Reilly-Knapp, M y Erskine R. G. (2003). Core concepts of an Integrative Transactional analysis [Conceptos nucleares de un Análisis Transaccional Integrativo]. Transactional Analysis Journal, 33(2), 168-177. https:// doi.org/10.1177/036215370303300208

Raynaudo, G. y Peralta, O. (2017). Cambio conceptual: una mirada desde las teorías de Piaget y Vygotsky. Liberabit, 23(1), 137-148. https://doi.org/10.24265/liberabit.2017.v23n1.10

Sánchez-Quintero, S. y De la Vega, I. (2013). Introducción al tratamiento basado en la mentalización para el trastorno límite de la personalidad. Acción Psicológica, 10(1), 21-32. http://dx.doi.org/10.5944/ap.10.1.7030

Turygin, N., Matson, J. L. y Adams, H. (2014). Prevalence of co-occurring disorders in a sample of adults with mild and moderate intellectual disabilities who reside in a residential treatment setting [Prevalencia de trastornos concurrentes en una muestra de adultos con discapacidad intelectual leve y moderada que residen en un entorno de tratamiento residencial]. Research in Developmental Disabilities, 35(7), 1802-1808. https://doi.org/10.1016/j.ridd.2014.01.027 